\title{
Systematic Study of Different Design Decisions in Markov Model-based Analysis of Molecular Structure Data
}

\author{
Sharmila Roychoudhury \\ Thomas Jefferson High School for Science and Technology
}

\begin{abstract}
Modeling and simulation software now provide us with a view of the structure space navigated by peptides and proteins under physiological conditions. Such software, such as Molecular Dynamics, yields trajectories of consecutive structures accessed by a dynamic molecule, but does not readily expose the underlying organization in the structure state so as to summarize the equilibrium dynamics over the present structural states. In this paper we investigate Markov State Models on their ability to do so. While we make use of an established software to do so, we analyze within it different design decisions and measure their impact on the obtained results. We present our findings on optimal design decisions, revealing in the process the dynamics of the Met-enkephaline peptide.
\end{abstract}

\section{CCS CONCEPTS}

- Applied computing $\rightarrow$ Molecular structural biology;

\section{KEYWORDS}

equilibrium structural dynamics, Markov state models

\section{ACM Reference Format:}

Sharmila Roychoudhury and Amarda Shehu. 2018. Systematic Study of Different Design Decisions in Markov Model-based Analysis of Molecular Structure Data. In ACM-BCB'18: 9th ACM International Conference on Bioinformatics, Computational Biology and Health Informatics, August 29September 1, 2018, Washington, DC, USA. ACM, New York, NY, USA, 3 pages. https://doi.org/10.1145/3233547.3233618

\section{INTRODUCTION}

We now know that biological molecules are intrinsically dynamic. Peptides and proteins, in particular, harness their structural equilibrium dynamics to interact with different molecular partners in the cell [1]. Molecular Dynamics (MD) simulations are increasingly providing us with a view of the structure space navigated by an amino-acid chain, one trajectory of structures at a time. Launching many such simulations on supercomputers yields many trajectories, but does not directly expose the underlying organization in the structure state so as to summarize the equilibrium dynamics over the present structural states. In response, a growing body of research utilizes Markov State Models (MSM), which analyze

\footnotetext{
${ }^{*}$ Corresponding Author: amarda@gmu.edu
}

Permission to make digital or hard copies of part or all of this work for personal or classroom use is granted without fee provided that copies are not made or distributed for profit or commercial advantage and that copies bear this notice and the full citation on the first page. Copyrights for third-party components of this work must be honored. For all other uses, contact the owner/author(s).

ACM-BCB'18, August 29-September 1, 2018, Washington, DC, USA

(C) 2018 Copyright held by the owner/author(s).

ACM ISBN 978-1-4503-5794-4/18/08.

https://doi.org/10.1145/3233547.3233618

\author{
Amarda Shehu* \\ Dept of Computer Science, George Mason University
}

trajectory data to constructed an MSM that summarizes the dynamics in terms of a few structural states [2]. The process requires many algorithmic components, executed serially. In this paper, we conduct a systematic evaluation of different design decisions in several of these components in order to reveal the most effective ones. We do so in the context of a simulation-based analysis of the Met-enkephaline (Met-Enk) peptide, for which we present the MSM summarizing its MD-probed dynamics.

\section{METHODS}

Our evaluation is conducted in the MSMBuilder [2] environment, which provides a pipeline with the following steps. (i) Provided one or more trajectories of structure data, a featurizer extracts the attributes of interest; many options are supported, such as dihedral angles or inter-atom distances. We select to proceed with dihedral angles to reduce the dimensionality of the data. (ii) The dimensionality is reduced even further by the dimensionality reduction step, which, by default, applies time-lagged Independent Component Analysis (tICA) [3] to the featurized trajectories. We evaluate an additional strategy, Principal Component Analysis (PCA) [3] in order to reveal the most effective one. (iii) The reduced/projected (structure) trajectories are then subjected to a clustering algorithm. We evaluate a comprehensive list of 7 clustering algorithms, evaluating their performance as a function of the number of clusters in order to determine both the most effective algorithm and the number of clusters corresponding to the identified (structural) macrostates. Lastly, we build the MSMBuilder with the most effective setting to show the state-to-state equilibrium dynamics for the Met-Enk peptide, for which a trajectory of 2,000 all-atom structures (collected every $1 \mathrm{ps}$ ) is obtained via an MD simulation at room temperature and in explicit solvent via the AMBER software. All our analysis was conducted in Python, utilizing the sklearn library.

\section{RESULTS}

\subsection{Comparison of Dimensionality Reduction}

After converted to dihedral angle-based representations via the featurizer, the data is subjected to PCA vs. tICA, and two results are related. The left panel of Figure 1 shows the accumulation of variance plot, which reveals that PCA captures more of the variance with fewer eigenvectors (components). However, the middle and right panels of Figure 1, which relates density plots over twodimensional projections of the data (considering all pairs over the top four components), shows that IICA projections capture more of the structural richness of the data, thus revealing more substates, than the PCA-based density plots. Taking it altogether, we proceed with tICA-based projections over all 16 components to reveal structural states that are emerging in these density plots. 

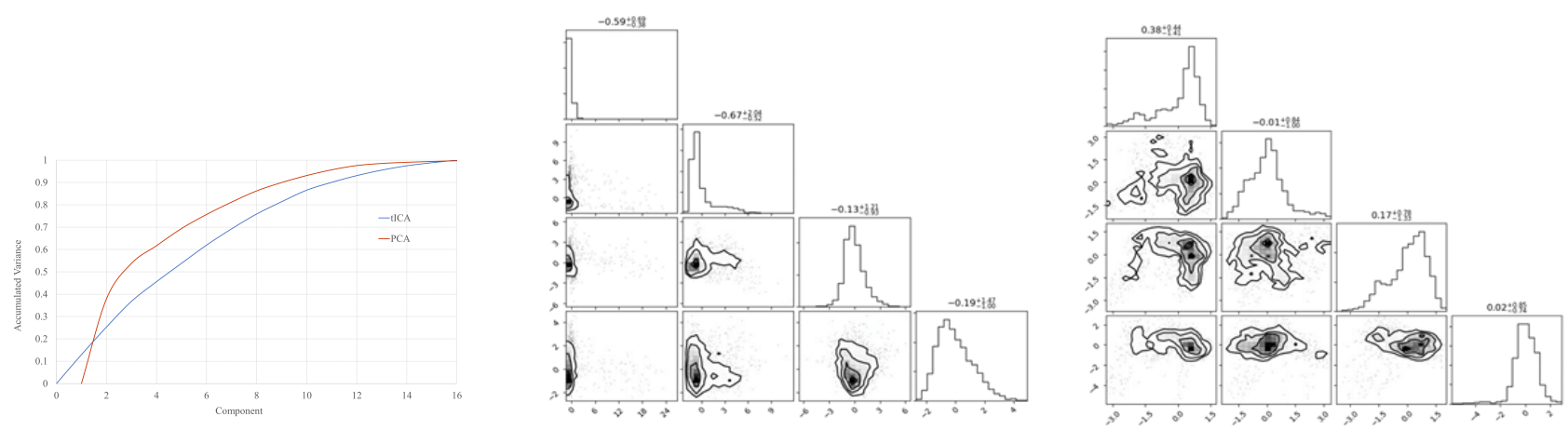

Figure 1: Accumulation of variance plots for PCA and tICA (left panel), followed by density plots of PCA (middle) versus tICA (right) projections.
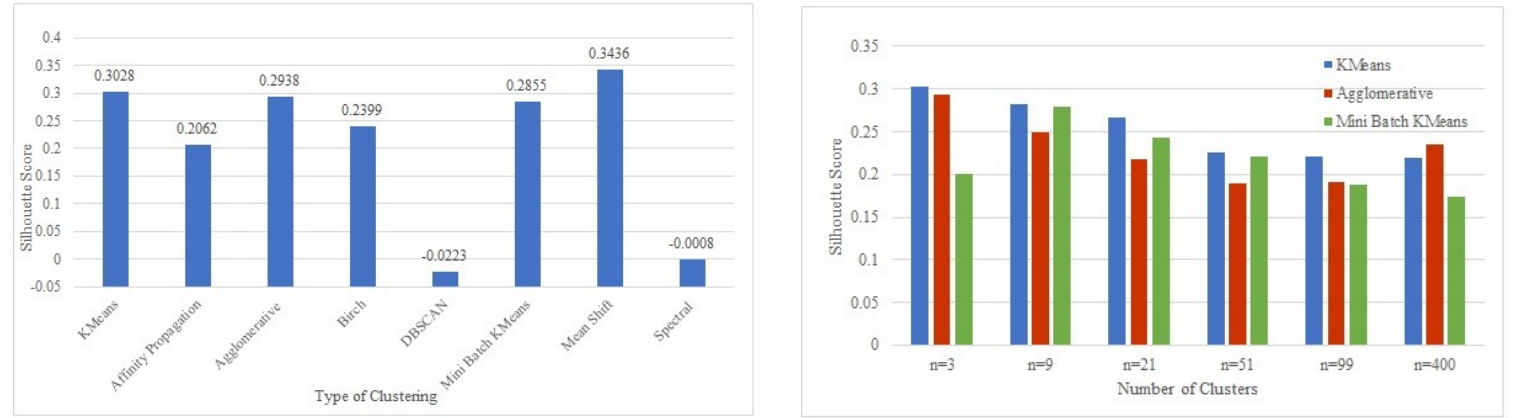

Figure 2: Left: Comparison via silhouette score of 7 clustering algorithms at $n=3$. Right: Comparison of top three clustering algorithms at varying $n$.

\subsection{Comparison of Clustering Algorithms}

Seven different clustering algorithms were applied onto the 16attribute data (corresponding to all-atom structures). The algorithms were evaluated via the silhouette score, fixing the number of clusters for algorithms dependent on such a specification, to 3, initially. This comparison is related in the top panel of Figure 2, which shows that K-Means, Agglomerative, and Mini Batch K-Means are the top three (achieving higher silhouette score over others). These three are further evaluated over a varying number of clusters, as related in the bottom panel in Figure 2, which shows K-Means with the number of clusters set to 3 as the better-performing one.

\subsection{Analysis of Built MSM}

The clustering results obtained via the top algorithm and setting identified above are then fed to the final step, the construction of the MSM, which is related in Figure 3: three identified states are equally long-lived, with self-transition probabilities ranging from 0.7 to 0.9 . Transitions between these states are rare (as evidence by low probabilities), pointing to a dynamics dominated by deep, stable states separated by energy barriers.

\section{CONCLUSION}

This study presents a computational protocol for evaluating design decisions in MSM-based analysis of MD structure data. In addition to revealing interesting findings on such decisions (such as K-Means providing the best clustering results), the protocol, applied to a dynamic peptide, whose functional plasticity remains poorly

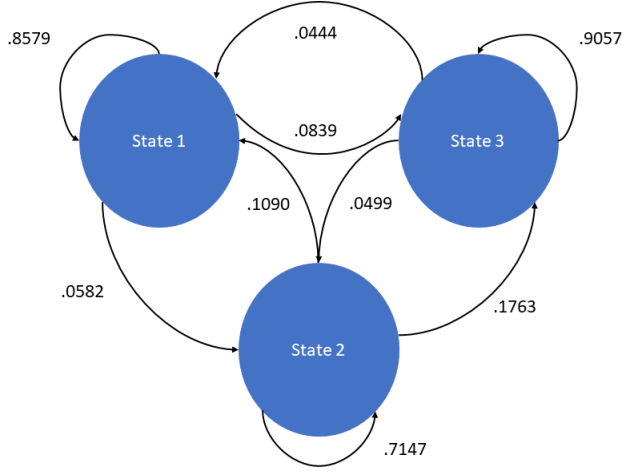

Figure 3: Schematic diagram of the obtained MSM.

understood, reveals a multi-state dynamics of possible value for understanding such plasticity.

\section{ACKNOWLEDGMENTS}

This work is supported in part by NSF IIS CAREER Award No. 1144106, a Jefferson Memorial Trust Award, and the Thomas Jefferson High School Partnership Fund.

\section{REFERENCES}

[1] D. D. Boehr, R. Nussinov, and P. E. Wright. 2009. The role of dynamic conformational ensembles in biomolecular recognition. Nature Chem Biol 5, 11 (2009), 789-96.

[2] M. P. Harrigan, M. M. Sultan, C. X. Hernández, and others. 2017. MSMBuilder: Statistical Models for Biomolecular Dynamics. Biophys 7 112, 1 (2017), 10-15.

[3] D. G. Luenberger. 1984. Linear and Nonlinear Programming (2nd ed.). AddisonWesley. 